\title{
El Maule como Prefrontera y Borde: De la postrimería imperial al Estado en forma, siglos XVIII-XIX'
}

\section{Martín Lara Ortega ${ }^{2}$ \\ Alfredo Gómez Alcorta ${ }^{3}$}

La historia del Maule es una historia compleja y difusa. Compleja, en la medida que carga con una historia de larga data, pues los asentamientos más antiguos del proceso de expansión español en Chile la tienen como testigo de aquello. Difusa, porque a pesar de los grandes esfuerzos por reconocerla como campo de estudio para quienes nos dedicamos al pasado, sigue siendo una mina de la que sólo se han mostrado pequeñas vetas de explotación (Sánchez, 2013 y 2015). Resulta singular reconocer que historiadores de la primera mitad del XX como Arnold Toynbee titulara uno de sus trabajos con el nombre de la región (1968), pero tanto para él como para nosotros, no era más que la excusa de reconocer en los espacios olvidados, ejercicios de búsqueda de cómo las singularidades espaciales e históricas se combinan y se proyectan en manifestaciones plurales $\mathrm{O}$, si se quiere, globales (Conrad, 2016: 45).

La presente exposición, que hasta este momento es una hipótesis de trabajo, pretende ofrecer una mirada panorámica y diacrónica de la región del Maule como una Prefrontera del imperio español en América (Herzog, 2015: 24). Partiendo de la premisa que el Maule conforma una de las múltiples fronteras históricas que existieron en el continente, mediante distintas voces daremos muestras de cómo se comprendió dicho espacio, considerando sus características, sus carencias y su permanente imagen de sumisión vertical entre sus habitantes y las autoridades primadas. Finalmente, plantearemos que el Estado ibérico y más tarde el nacional, mediante una serie de estrategias de racionalización de recursos, sumado a una susceptible condición

1 Este texto fue presentado originalmente bajo el título "Borde y olvido: El Maule como Prefrontera. Siglos XVII-XIX", en el Seminario Fronteras y nuevas fronteras. Discursos, representaciones y perspectivas de análisis. Siglo XVI-XIX, realizado el 26-IX-2016, en el Departamento de Historia de la Universidad Adolfo Ibáñez, Chile.

2 Chileno. Magíster en Historia, Pontificia Universidad Católica de Chile. Académico, Universidad Autónoma de Chile. E-mail: martinlara@uc.cl

3 Chileno. Magíster en Artes y Humanidades con mención en Historia, Universidad Gabriela Mistral, Chile. Académico, Universidad Bernardo O'Higgins, Chile. E-mail: alfredo.gomezalcorta@gmail.com 
de abandono, darán entre los habitantes del Maule la imagen de su región como un borde olvidado del mundo civilizado.

De la misma forma, esta presentación nos permitirá teorizar alternativas conceptuales sobre los estudios fronterizos, en donde Prefrontera y Borde, más cercanos a la disciplina geográfica que a la Historia, pueden aportar precisiones para comprender temas conocidos de la historiografía nacional.

Recientemente Sebastián Conrad, profesor de la Freie Universität Berlín, publicó What is Global History?, que en su preámbulo nos invita a no sectorizar para comprender ni comparar para trazar ideas, sino que su propuesta apunta a que el historiador global debe pensar en términos de integración por sobre la conexión, mirando atentamente las dinámicas que conectan las piezas de aquel rompecabezas que llamamos "pasado" y que en términos espaciales se transforma en un escurridizo y a veces complejo juego de ajedrez. Sobre esta propuesta, el Maule si bien comprendemos que es único en la medida que es una región singular e irrepetible; entendemos también que la sociedad que dinamizó su historia, carga con un conjunto de valores y mentalidades susceptibles de ser analizados bajo parámetros culturales, políticos y económicos de naturaleza global o, si se quiere, occidental.

\section{Maule como Prefrontera}

La historiografía nacional, atenta receptora de las tradiciones europeas y norteamericanas, ha tenido la posibilidad de tomar y apropiarse de diversas líneas de investigación, siendo el tema de la "frontera" uno de los más fecundos durante la segunda mitad del siglo pasado. Los trabajos de Mario Góngora (1966), Sergio Villalobos (1995), Leonardo León (2003) y más recientemente de Ximena Urbina (2009), han dado luces no sólo de la importancia de la idea de frontera como garante de un ideal nacional, sino también de cómo este conformó a las sociedades que los habitaban. Claramente estoy haciendo referencia a lo sucedido en la propiamente "frontera araucana", en donde conceptos como Flandes Indiano, Marca Araucana, Confín y Limes imperial (Mantecón \& Truchuelo, 2016), recrean un imaginario que hace recordar viejas lecturas y persistentes ideas que de un modo $u$ otro, siguen girando en el actual debate nacional. Pero, si bien aquellos trabajos tienen tras de sí haber sido la vanguardia del debate en nuestro campo de investigación, sabemos también que las vanguardias son las primeras en caer en el campo de batalla.

Nuestra propuesta es escudriñar, haciendo propia la descripción de este seminario, que "los espacios americanos se han definido y redefinido constantemente a partir de tensiones, fricciones, adopciones y traducciones de los diferentes idearios". Apropiándonos de aquella "traducción" a que nos desafía la profesora Macarena Cordero en la introducción de esta reunión, 
quiero invitarlos a pensar sobre las prefronteras americanas como una alternativa de investigación, como un espacio rico de sintomatologías históricas, donde podemos encontrar los vestigios y antecedentes no solamente del comportamiento social de los habitantes de aquel espacio, sino cómo se entienden las dinámicas económicas y políticas imperiales y más tarde nacionales que se fueron gestando. Trabajos como los de Miguel Olmos en México (2013), quien ha contribuido a cuestionar la actual concepción de frontera chicana entre Estados Unidos y México para retroceder a los viejos espacios de California y Texas en vísperas de la ocupación norteamericana, entendiéndolos en la práctica como una Prefrontera; o los trabajos de nuestra colega Ingrid de Jong en Argentina, quien en sucesivos trabajos ha deshilvanado las complejas relaciones de poder entre comunidades indígenas segmentadas de la Patagonia y el Estado nacional argentino (De Jong, 2014: 155), son avances en esa línea, y nosotros queremos proponer aquí nuevas alternativas de entendimiento y reflexión sobre este tradicional tema de estudio.

Sobre estos esfuerzos, Prefrontera y Borde se transforman en una díada conceptual dinámica y voluble que hace del Maule un espacio privilegiado de análisis. El Maule para nosotros es atractivo en la medida que es un espacio lleno de claroscuros y contrariedades, realidad analítica irresistible para el historiador. Si bien está habitado hacia el siglo XVIII, la dimensión espacial es excesivamente amplia y dispersa, haciendo que la población desaparezca entre el monte andino y las templadas zonas costeras. Ahora bien, esta población primitiva con las políticas imperiales de poblamiento, ve converger en el Maule a nuevos habitantes que en su gran mayoría provienen de las profundidades de la frontera araucana. Tengo entendido que el profesor Chuecas (2016) ha dado luces de aquellas migraciones a las inmediaciones de la ribera norte del Biobío, situación que yo extiendo más allá del Itata y Nuble, llegando incluso al confín norte de la intendencia de Concepción, en la denominada Isla del Maule.

De esta forma, lo primero que se puede intuir entre los contertulios, es que la región del Maule es una Prefrontera no solo por anteceder espacialmente el espacio entre Santiago y Concepción, denotando en dicho espacio características que si bien no son de choque cultural e intercambios simbólicos, que sí se dan en la frontera; en el Maule serán otras las características que dinamizan los imaginarios de aquellos habitantes. Para ser preciso, la idea de olvido por parte de las autoridades en relación a apoyo material y presencia factual, sumado a ser considerada sólo como una región de paso o tránsito, irán conformando en el ethos maulino una idea de abandono y marginalidad de sus intereses durante el imperio y más tarde la república.

Desde otro punto de análisis, el Maule adquiere características históricas de Prefrontera en la medida que se van entretejiendo políticas imperiales de 
poblamiento que hacen que la Frontera alimente con población y tradición cultural a esta Prefrontera. En otras palabras, el Maule y Araucanía están vinculados por profundas políticas ibéricas que permitieron generar una historia común, en donde la población, unida por lazos familiares, tradición cultural y virtual concepción de pertenencia a un Estado, son elementos comunes. Por sólo dar un testimonio que grafique aquello, lo da nuestra exploración demográfica sobre persistencia de apellidos en el Maule sur, en donde se denota un $90 \%$ de coincidencia con los apellidos canónicamente fronterizos, repitiéndose los Fuentes, Sepúlveda y Hernández (Lara, 2016), siendo de resultado similar a los que han evidenciado anteriormente los trabajos de Sergio Villalobos y Cristián Rodríguez (1997).

El Maule durante el siglo XVIII, como ya adelantamos, es un espacio de circulación y de tránsito constante al ser un territorio intermedio entre la capital de la gobernación y la ciudad de Concepción (Lara, 2013: 18). Pero, paradójicamente, también tiene perfumes propios de Finis Terrae, en la medida que sus comarcas eran la zona límite de las dos intendencias coloniales.

Esta Prefrontera en el cambio de siglo XVIII-XIX vivió fuertemente las tribulaciones del proceso de independencia. En el debate político que ocupaba a los vecinos de Talca, Linares y Parral por consolidar una salida realista o patriota, permitió que el vacío de poder político y el miedo fueran factores de significativa ambivalencia en torno a la posición política que las circunstancias obligaban a tomar. Casos como el cura parralino José Urrutia o el hacendado Ángel Calvo de Talca, quienes decididamente tomaron partido por el lado realista, son prueba de aquello (Barros Arana, 2002). Ahora bien, creemos que la decisión de tomar un camino u otro, va más allá de las circunstancias bélicas, sino que tiene un trasfondo social y político que se basa en qué bando daba las mejores garantías de gobierno y atención a las necesidades de los "vecinos" de la región.

Desde otra perspectiva, y ya avanzado el establecimiento realista en Chile entre 1814 y 1817, el desarrollo de dicha confrontación dio lugar a convertir al Maule en un espacio de cobijo para algunas de las familias patricias provenientes de Santiago que durante la reconquista, junto a sus esclavos de servicio, tuvieron en estas tierras algo de tranquilidad, cierta estabilidad y nuevos espacios de desenvolvimiento social (Medina, 1900: 286). De hecho, a pesar de recientes trabajos recopilatorios como los de Carolina González (2014) y Jaime Valenzuela (2017), aun poco y nada se sabe qué pasó con los negros y afrodescendientes maulinos tras la abolición de la esclavitud, permitiendo preguntarnos: ¿huyeron al norte? ¿Volvieron al Perú? ¿Se mezclaron con los habitantes locales generando descendencia hasta nuestros días? Otro tema aún por trabajar. 


\section{Maule como Borde}

Ya la idea de Frontera como concepto de trabajo, si bien no se puede desechar, queda disminuida en desmedro de otras como Borde, otro concepto de la gran familia temática. En su primera acepción "Extremo u orilla de algo" (RAE, 2016), creemos que es el mejor concepto que podría identificar o reflejar las voces de los maulinos en el trayecto de los años imperiales a republicanos.

Sólo aquellos lugares que tienen esas características de Borde, son los que pueden testimoniar de mejor forma la convergencia de violencia, poder y justicia (2016) que enmarcados en la fuerza de ordenamiento y verticalidad de mando, son el escenario propicio para actuar. Por muy lejos que Madrid se encuentre del Maule, su poder lo hace sentir y transmitir. Ambrosio O'Higgins, quien entre 1786 y 1788 actuó como intendente de Concepción, junto con recorrer en las clásicas visitas palmo a palmo las tierras de su intendencia, con el mismo ímpetu hizo cumplir la ley cuando se la infringía, como sucedió -según cuenta Ricardo Donoso- con un sujeto que mando a descuartizar y enviar cada cuarto a los cuatro confines de la intendencia (1941).

Durante el siglo XIX, la sociedad del Maule hace extremas sus consideraciones sobre sí misma, que se ven de manera manifiesta en sus cartas a las autoridades santiaguinas. A esta hora cercana al almuerzo, no tengo intención de aburrirlos con citas tras citas de vecinos que apelan por apoyo material, menos en vísperas de elecciones municipales, pero sí decantar las ideas que tras dichas demandas comienzan a aparecer. En una serie de documentos que se concentran en la década de 1870 se insiste que el Maule y las zonas circundantes a las villas más conocidas son zonas que están abandonadas a su suerte, en donde la violencia en su más amplio espectro se toma las calles y campos de la región. Alcoholismo, abusos sexuales, violencia de género, tienden a aparecer de manera más concentrada y vehemente, cuestionando finalmente la eficiencia del Estado. El poder social, íntimamente vinculado al poder político, genera tensiones sociales que en la prensa tienen su punto de eyección. La modernidad con su diafanía informativa, ya sea liberal o conservadora, genera relatos de caos social y desestabilidad de una jerarquía (Stuven, 2011). Evidentemente, si bien existen culpables fácticos de todo lo que acontece, sin dudas para los maulinos es el Estado con sus funcionarios y agencias los principales culpables. Nuevamente el Maule se asemeja más a una proyección cartográfica de Ortelius rodeada por monstruos marinos, que al esfuerzo normativizador que el Estado nación quiere impulsar.

Al revisar la prensa de la ciudad de Parral en el último cuarto del siglo XIX que, intuimos, debe ser similar a la de otras villas y ciudades maulinas, podemos observar el constante reclamo de los vecinos hacia las autoridades 
por la falta de su presencia, gestión y recursos para dar solución a los problemas sociales y catástrofes naturales que se dan. Por ejemplo, en 1870 el diario El Iris en su columna de noticias locales presentaba este macabro relato:

"El miércoles en la noche fue asaltada por una partida de asaltadores la casa de campo de la señora doña Valentina Gutiérrez, habiéndole desarrojado las puertas, se introdujeron al interior de sus habitaciones donde ella y demás familia dormían. Allí, vendándoles la cara, dieron principio por torturar de un modo horrible a la señora, colgándola en las vigas por tres veces y azotándola bárbaramente con el fin de que dijese dónde tenía el dinero. Estos crímenes en la provincia son un mal crónico que proviene de la mala elección de los subdelegados e inspectores, pues siempre estos destinos son sufridos por personas de inteligencia y energía para perseguir a los malhechores; y muchas veces ellos mismos patrocinan a este tipo de gente, que se pasean libremente infundiendo el terror en nuestros campos" (El Iris, 20-II-1870).

Aquí tenemos la oportunidad de darle proyección histórica al concepto de Borde, en la medida que Parral como ciudad que era penetrada simbólicamente por el mundo rural y viceversa, es un reflejo sintomático de lugares intermedios entre caos y orden $\mathrm{o}$, si se quiere, civilización y barbarie. Aquel frágil equilibrio o punto intermedio se convierte nominalmente en Borde. Con este ejemplo, queremos afirmar que dicho concepto permite situar al historiador en un punto de análisis más preciso que el de frontera, el cual creemos que si bien tiene un universo abarcable en términos analíticos, es muy grueso para las precisiones de observación de un determinado problema como el que acabamos de testimoniar. En definitiva, para esta ocasión, Borde se transforma en un concepto que precisa un subconjunto de realidades históricas que escapan a la proyección mayor que da el estudio del clásico concepto de frontera.

Desde otra dimensión, en donde se expresa el olvido de las autoridades hacia los maulinos, se da desde la insuficiente presencia del tercer poder del Estado, el judicial. Aquí vemos cómo se retrotraen las viejas formas de hacer justicia de la colonia con el descuartizamiento, da paso a una lenta espera de un juicio para dirimir las culpabilidades y condenas definitivas de los transgresores. Al respecto, creo necesario mencionar que en los últimos años en América Latina y en Chile, se ha venido gestando un florecimiento de dicha temática que ha tenido en autores como los integrantes del Grupo de Estudios Historia y Justicia unos activos promotores de dicha línea de trabajo. Dentro de aquel colectivo, nuestro colega y amigo Víctor Brangier desde hace unos años ha venido trabajando el tema, aplicando al caso del Maule determinadas propuestas, en donde reconoce que uno de los princi- 
pales elementos que dibuja la constitución del Estado en forma, fue la falta de presencia de la justicia letrada en los departamentos provinciales. Esto en la provincia del Maule, en particular en la ciudad de Cauquenes, como ciudad cabecera de la provincia, hizo que muchos jueces legos, inspectores, subdelegados y alcaldes de ciudades como Linares y Loncomilla, sobrepasaran sus propias atribuciones en beneficio de dar orden y hacer justicia para tranquilidad de los vecinos. Sin embargo, dicha acción, en observación del poder judicial, eran actos y decisiones que no los facultaba la constitución de 1833, que a través de sus leyes y normativas, señalaba que era función exclusiva de los jueces de letras la aplicación y sentencia penal de los condenados (Brangier, 2013: 8-10).

\section{Consideraciones finales}

Con todo lo que hemos dicho en estos breves minutos, planteamos en términos históricos, que el Estado ibérico y más tarde el nacional, mediante una serie de estrategias, no pudieron generar en el imaginario maulino una idea de centralidad, integración o de participación política en un cuadro mayor 0 , si se quiere, nacional. De las formas ejemplares de impartir justicia en el período indiano, hasta llegar al abandono excesivo que reclaman los vecinos en la década del 70' del siglo XIX, nos permitimos interpretar el pensamiento de los habitantes del Maule sobre que su región era un borde olvidado del mundo civilizado. En otras palabras, barbarie entre la civilizada Santiago y la caótica frontera araucana.

En términos conceptuales, como reza la invitación a este seminario, Prefrontera y Borde son ideas conformantes de una "pluralidad de direcciones e interpretaciones complejas, complementarias y a la vez contradictorias entre sí". En este caso, quisimos aplicar estos dos conceptos como una alternativa para la disección del pasado histórico de la región del Maule. Con la utilización de aquella díada, pensamos que los trabajos temáticos aplicados a dicho territorio adquieren la vitalidad de un espacio de detención y observación no solo para los historiadores, sino para los literatos y estudiosos de las humanidades en general que, como dijimos al inicio de la presentación, se convierte en una rica mina lista para ser explotada. 


\title{
Referencias Bibliográficas
}

\section{Fuentes Primarias}

\author{
a) Periódicos \\ El Iris, Parral, 1870.
}

b) Impresos

Medina, J. T. (1900). Colección de Historiadores y Documentos Relativos a la Independencia de Chile, Tomo V. Santiago: Imprenta Cervantes.

\section{Fuentes Secundarias}

a) Artículos

Brangier, V. (2013). "Tensiones en el seno de la esfera judicial letrada: las Visitas Judiciales de la Corte de Apelaciones de Concepción hacia los jueces de letras de la Provincia de Maule", en XIV Jornadas Interescuelas/Departamentos de Historia. Mendoza: Departamento de Historia de la Facultad de Filosofía y Letras, Universidad Nacional de Cuyo.

De Jong, I. (2014). "Prácticas estatales sobre una sociedad segmental: La subordinación militar de los indios amigos en Azul y Tapalqué (1850-1870)", en TEFROS, Vol. 12, № 2.

Lara, M. (2013). "Historicidad del Maule. Génesis de una región", en SudHistoria, № 7 .

Mantecón, T. y S. Truchuelo (2016). "La(s) frontera(s) exteriores e interiores de la Monarquía Hispánica: perspectivas historiográficas", en Historia crítica, Nㅜ 59.

Villalobos, S.; Rodríguez, C. (1997). "El espacio rural Longaví-Núuble 1737", en Cuadernos de Historia № 17.

\section{b) Libros}

Barros Arana, D. (2002). Historia general de Chile. Tomo IX. Santiago: DIBAMEditorial Universitaria.

Conrad, S. (2016). What is Global History? Princeton: Princeton University Press.

Donoso, R. (1941). El Marqués de Osorno Don Ambrosio O'Higgins: 1720-1801. Santiago: Universidad de Chile. 
Góngora, M. (1966). Vagabundaje y sociedad fronteriza en Chile (siglos XVII a $X I X)$. Santiago: Facultad de Ciencias Económicas, Universidad de Chile.

González C. (2014). Esclavos y esclavas demandando justicia. Chile, 1740-1823. Documentación judicial por carta de libertad y papel de venta. Santiago: Fondo Juvenal Hernández Jaque, Universidad de Chile-Editorial Universitaria.

Herzog, T. (2015). For Frontiers of Possession: Spain and Portugal in Europe and the Americas. Cambridge: Harvard University Press.

León, L. [Et Al.] (2003). Araucanía: La frontera mestiza, siglo XIX. Santiago: Ediciones Universidad Católica Silva Henríquez.

Olmos, M. (2013). Antropología de las fronteras. Alteridad, historia e identidad más allá de la línea. Tijuana: El Colegio de la Frontera Norte.

Sánchez R. (2015). Agua y patrimonio en la región del Maule (1850-1930). Una mirada desde la historia. Talca: Consejo Nacional de la Cultura y las Artes, Universidad Autónoma de Chile.

Sánchez R. (2013). Por la libertad de pensamiento: La educación laica en Talca (1742-2013). Talca: S.I.

Stuven, A. M. (2011). La seducción de un orden. Santiago: Ediciones Pontificia Universidad Católica de Chile.

Toynbee, A. (1968). Entre el Maule y el Amazonas. Buenos Aires: Editorial Francisco de Aguirre.

Urbina, M. X. (2009). La frontera de arriba en Chile colonial: Interacción hispanoindígena en el territorio entre Valdivia y Chiloé e imaginario de sus bordes geográficos 1600-1800. Valparaíso: Ediciones Universitarias de Valparaíso.

Valenzuela J. (2017). América en diásporas: esclavitudes y migraciones forzadas en Chile y otras regiones americanas (siglos XVI-XIX). Santiago: RIL Editores-Instituto de Historia, Pontificia Universidad Católica de Chile.

Villalobos, S. (1995). Vida fronteriza en la Araucanía: El mito de la guerra de Arauco. Barcelona: Editorial Andrés Bello.

\section{c) Tesis}

Chuecas, I. (2016). Dueños de la frontera. Terratenientes y sociedad colonial en la periferia chilena. Isla de La Laja, 1670-1845. Tesis de doctorado en Historia (inédita). Santiago: Pontificia Universidad Católica de Chile.

Lara, M. (2016). Historia de la vida cotidiana en Parral 1870-1880. Tesis de maestría en Historia (inédita). Santiago: Pontificia Universidad Católica de Chile. 